\title{
The NIH Blueprint for Neuroscience Research Seeks Community Input on Future Neuroscience Investments
}

\author{
Meghan C. Mott, Christopher P. Austin, Diana W. Bianchi, Ann K. Cashion, Joshua A. Gordon, Jill E. Heemskerk,

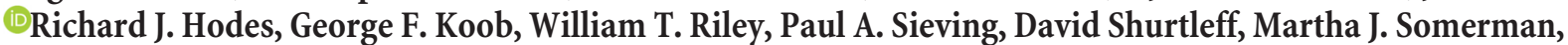 \\ - Nora D. Volkow, Kathleen C. Anderson, David F. Owens, and $\odot$ Walter J. Koroshetz
}

The NIH Blueprint for Neuroscience Research, a group of 14 NIH Institutes, Centers, and Offices (ICOs) that support research on the nervous system, recently released a Request for Information (https:// grants.nih.gov/grants/guide/notice-files/ NOT-NS-19-002.html) on the Proposed Funding Priorities for Neuroscience Research and Input on Cross-Cutting Opportunities. This RFI seeks input on future Blueprint activities and investments that have the potential to make the broadest possible impact for the neuroscience community. Ideally, suggestions should take into consideration how the activities proposed would complement other ongoing $\mathrm{NIH}$ neuroscience research, such as the NIH BRAIN Initiative (https:// www.braininitiative.nih.gov/) and programs supported by the participating Blueprint ICOs.

Neuroscience research is a unifying theme across many NIH ICOs. The NIH Blueprint for Neuroscience Research (Blueprint; https://neuroscienceblueprint.nih. gov/) was established in 2004 as a collaborative framework that includes the $\mathrm{NIH}$ Office of the Director and 13 NIH Institutes and Centers to broadly advance the area of neuroscience. By coordinating research, training, tool, and resource development in cross-cutting areas of neuroscience, Blueprint ICOs confront challenges that are too large for any single ICO.

Over the last 14 years, Blueprint has established unique training opportunities for individuals from diverse backgrounds underrepresented in neuroscience research,

Received 0ct. 19, 2018; revised 0ct. 26, 2018; accepted 0ct. 27, 2018.

Correspondence should be addressed to Meghan C. Mott at mottmc@ninds.nih.gov.

https://doi.org/10.1523/JNEUROSCI.2742-18.2018

Copyright $\odot 2019$ the authors $\quad 0270-6474 / 19 / 390774-02 \$ 15.00 / 0$ such as the Enhancing Neuroscience Diversity through Undergraduate Research Education Experiences (ENDURE; https:// neuroscienceblueprint.nih.gov/endureundergraduate-education) program and the Diversity Specialized Predoctoral to Postdoctoral Advancement in Neuroscience (D-SPAN F99/K00 Award; https:// neuroscienceblueprint.nih.gov/nihblueprint-D-span-award-f99k00) program. From 2011 to 2016 Blueprint funded four training programs in computational neuroscience that provided training in both experimental neuroscience and theories and principles of the physical, computer, mathematical, or engineering sciences that encouraged students in the quantitative sciences to pursue neuroscience research early in their careers and students in the biological/behavioral sciences to become educated in quantitative sciences (https://neuroscienceblueprint. nih.gov/training/training-computationalneuroscience).

Blueprint has also developed research resources and tools to assist neuroscientists such as the NIH Toolbox for assessment of cognitive, emotional, sensory, and motor functions (http://www.healthmeasures.net/exploremeasurement-systems/nih-toolbox), the neuroimaging tools and resources collaboratory (NITRC; https://www.nitrc.org/), the neuroscience information framework (NIF; https://neuinfo.org/), and the nonhuman primate (NHP) brain atlas of the developing rhesus macaque brain (https://neuroscienceblueprint.nih.gov/resources-tools/ non-human-primate-brain-atlas). Beginning in 2014, Blueprint made substantial investments in The NIH BRAIN Initiative, which continue to accelerate the development of innovative neurotechnologies to produce a dynamic picture of the brain and how its neural circuits interact.
Blueprint also launched a series of Grand Challenges to catalyze research with the potential to transform our basic understanding of the brain and our approaches to treating brain disorders. These included the Human Connectome Project (HCP), an ambitious effort to map all the connections of the human brain, which today provides an unparalleled compilation of neural data and an interface to graphically navigate these data to better understand complex brain pathways. This monumental task could be made possible only through the development of powerful new tools and technologies generated by advances in computer science, mathematics, and imaging and data visualization. HCP enlisted a diverse entourage of researchers-biologists, physicians, computer scientists, and physicists - at many institutions across the country. The technical advances achieved by the project have transformed the field by creating a standard in the brain imaging community that allows neuroscientists to aggregate data in unprecedented ways. HCP launched the second phase of the program by creating the Lifespan Connectome in 2015 which extended data collection from healthy subjects of all ages (https://neuroscienceblueprint.nih.gov/ Human-Connectome/connectome-projects? combine $=$ \&field_connectome_category_ tid $\% 5 \mathrm{~B} \% 5 \mathrm{D}=94$ ). Altogether these projects will map the long-distance brain connections and their variability in $>3000$ children and adults across the United States. HCP is paving the way toward a detailed understanding of how our brain circuitry changes as we age and how it differs in psychiatric and neurologic illness. Another Grand Challenge includes the Blueprint Neurotherapeutics Network, which was designed to address the lack of effective treatments for nervous system disorders by providing funding 
and resources to facilitate small molecule drug discovery and development to derisk potential therapeutics to the point that industry will invest in them. The BPN provides neuroscience researchers with funding and access to a full range of industry-style drug development services and expertise and is intended for projects requiring medicinal chemistry optimization and contract research organization support through phase I clinical testing. Since 2010, BPN has awarded 22 grants across the discovery, preclinical, and early clinical stages to develop novel therapeutics in Alzheimer's disease, hearing loss, smoking cessation, dry age-related macular degeneration, and migraine. Many of BPNfunded projects have attracted outside commercial partners including AstraZeneca, Trevena, Life Sciences Discovery Fund, Lin BioScience, BlackThorn Therapeutics, and Johnson \& Johnson. Finally, the Blueprint Grand Challenge in Chronic Neuropathic Pain supported research to understand the changes in the nervous system that cause acute, temporary pain to become chronic. It supported multi-investigator projects partnering researchers in the pain field with researchers in neuroplasticity resulting in $>80$ publications related to pain and neural plasticity.

Recently, Blueprint developed three Funding Opportunity Announcements that will support research beginning in FY2018. The Dynamic Neuroimmune Interactions in the Transition from Normal CNS Function to Disorders program seeks to transform our understanding of the dynamic changes among multiple neuroimmune components and how they contribute to the onset and progression of
CNS disorders. Research supported by this program is expected to address temporal changes in multiple neuroimmune components, such as neurons, microglia, and astrocytes. The Innovative Approaches or Technologies to Investigate Regional, Structural, and Functional Heterogeneity of CNS Small Blood and Lymphatic Vessels program solicited research focused on the development of new technology and tools or novel mechanistic studies (or a combination of both) to image, profile, and map CNS small blood and lymphatic vessels in health and disease across the lifespan. Additional goals of this research are to elucidate mechanisms underlying CNS small blood and lymphatic vessels structure and functional heterogeneity, differential susceptibility to injury, role in disease and repair processes, and responses to therapies. The Development and Validation of Technologies for Rapid Isolation and Characterization of Extracellular Vesicles (EVs) of CNS Origin program is a phased innovation award focused on technology development for robust and reproducible CNS-EV isolation methods. The primary goal of this initiative is to advance the current technologies, develop novel techniques and approaches, and standardize protocols to reliably and specifically isolate EVs of CNS origin from human biofluids, identify the cell type from which they were derived, and characterize their composition.

Many of the activities and research programs enumerated here were developed in response to input that $\mathrm{NIH}$ received directly from the scientific community. As Blueprint begins to plan the next phase of investments, we have released a Request for Information on Proposed Funding Priorities for Neuroscience Research and Input on Crosscutting Opportunities (https://grants.nih. gov/grants/guide/notice-files/NOT-NS-19002.html). As the Blueprint ICOs take stock of previous and ongoing programs, we are interested in new ideas about how to ensure sustained progress in neuroscience research across myriad diseases, disorders, and Institute missions. We are interested in ideas that are broadly relevant to all research on the nervous system (and not just relevant to the scientific portfolio of individual ICOs), particularly research that has the potential to transform our basic understanding of the brain and our approaches to treating nervous system disorders. We are also interested in ideas for innovate neuroscience training, research resources, infrastructure, and workforce development. Responses to this RFI should suggest how Blueprint could have the broadest possible impact for the neuroscience community in a value-added fashion over the current activities of the participating Blueprint ICOs. Are there infrastructure or research resources needs that cut across all of neuroscience? For example, should NIH expand on programs in developmental and/or computational neuroscience, the dissemination of neuroscience tools or supporting core facilities? How could $\mathrm{NIH}$ enhance neuroscience training and workforce diversity in the future? Your input is crucial; help shape the future of the NIH Blueprint for Neuroscience Research by submitting your input, which will be considered through May 2019. All comments must be submitted electronically to: https://neuroscienceblueprint.nih. gov/rfi-blueprint-webform. 